\title{
Shock-wave model of the earthquake and Poincaré quantum theorem give an insight into the aftershock physics.
}

\author{
Vladimir Kuznetsov \\ Institute of cosmophysical research and radiowave propagation FEB RAS \\ 684034 Kamchatka, Russia ${ }^{1}$
}

\begin{abstract}
A fundamentally new model of aftershocks evident from the shock-wave model of the earthquake and Poincaré Recurrence Theorem [H. Poincare, Acta Mathematica 13, 1 (1890)] is proposed here. The authors (Recurrences in an isolated quantum many-body system, Science 2018) argue that the theorem should be formulated as "Complex systems return almost exactly into their initial state". For the first time, this recurrence theorem has been demonstrated with complex quantum multi-particle systems. Our shock-wave model of an earthquake proceeds from the quantum entanglement of protons in hydrogen bonds of lithosphere material. Clearly aftershocks are quantum phenomena which mechanism follows the recurrence theorem.
\end{abstract}

Key words: quantum Poincaré recurrence theorem, shock-wave model of an earthquake, aftershock physics.

\section{Introduction}

An extremely interesting article devoted to the proof of the Poincare Recurrence theorem for quantum systems opening the way to solving a number of problems related to the physics of quantum entangled (QE) objects was published in February, 2018 [1]. Besides it another problem, for example, provides causes of resonances in the Solar system, etc. This theorem can be useful in solving problems related to the participation in them of many-particle quantum systems (MQS).

The shock-wave model of an earthquake [2,6] implies the presence of such ISS in the medium [3,4]. Only a quantum coupled system provides the appearance of a shock wave in a huge earthquake source. For example, for the strongest earthquakes with magnitude $M \approx 9$ the linear size of the earthquake source $L$ is $1,000 \mathrm{~km}$. As the earthquake synchronization otherwise, is the spread in times of the first arrivals at seismographs, $(t)$ is near 1 second, the synchronization rate is about $(L / t) \approx 10^{3} \mathrm{~km} / \mathrm{s}$, it brings the problem of an earthquake physics from the theory of elasticity and to the field, as Einstein once called, of "terrible long-range", which is typical for quantum entangled systems. Thus, approaches in QS used in the shock-wave model of an earthquake and, of course, in physics of their aftershocks are reasonable. Aftershocks are usually recorded in the same environment as the earthquake that causes them. Here, the author develops his assumption that a similar aftershock can occur in a completely different environment, on condition that it is quantum entangled with lithosphere. For example, if a shock crystallization occurs in supercooled water of a cloud then a shock wave of crystallization is generated [5] and its aftershocks are not earthquake-related. An example of quantum entanglement between supercooled water and an alcoholic solution of camphor is illustrated by stormglass, in which the growth of camphor crystals synchronizes with crystallization of supercooled clouds quantum entangled with it [7].

\section{Poincare's recurrence theorem.}

Let $g$ be a measure-preserving continuous one-to-one transformation, mapping the bounded set $D$ of Euclidean space into itself: $g D=D$. some $n>0$.

Then in any neighborhood $U$ of any point of the domain $D$ there is a point $x \in U$ that returns to $U ; g^{n} x \in U$ for

This theorem [8] is applicable, for example, to the phase flow $g^{t}$ of a two-dimensional system with potential $U\left(x_{1}, x_{2}\right)$ growing at infinity; in this case the invariant bounded domain in the phase space is given by the condition

$$
D=\{p, q: T+U \leq E\} .
$$

The Poincaré recurrence theorem can be strengthened by proving that almost every moving point repeatedly returns to its original position. This is one of the few general conclusions about the nature of the movement. Details of the movement are not known to anybody even in the case

$$
\ddot{x}=-\frac{\partial U}{\partial x}, x=\left(x_{1}, x_{2}\right)
$$

A somewhat paradoxical conclusion from the theorems of Poincaré and Liouville is the following prediction: if we open the partition separating the chamber with the gas and the vacuum chamber, then after a while the gas molecules

${ }^{1}$ e-mail: vvkuz38@mail.ru 
will almost certainly be reassembled in the first chamber (Fig. 1-A). The solution of the paradox is that "some time" is longer than the time of the existence of the solar system.

\subsection{Proof of Poincare's theorem}

Consider the images of a neighborhood $\boldsymbol{U}$ (Рис. 1-В):

$$
U, g U, g^{2} U, \ldots . g^{n} U, \ldots
$$

All of them have the same positive volume. If they did not intersect, the volume $\boldsymbol{D}$ would be infinite. Therefore, for some $\boldsymbol{k} \geq \mathbf{0}, \boldsymbol{l} \geq \mathbf{0}, \boldsymbol{k}>\boldsymbol{l}$

$$
\boldsymbol{g}^{k} \boldsymbol{U} \cap \boldsymbol{g}^{l} \boldsymbol{U} \neq \mathbf{0}
$$

Consequently, $\boldsymbol{g}^{\boldsymbol{k}-\boldsymbol{l}} \boldsymbol{U} \cap \boldsymbol{U} \neq \mathbf{0}$. Let $\boldsymbol{g}^{\boldsymbol{k}-l} \boldsymbol{x}=\boldsymbol{y}, \boldsymbol{x} \in \boldsymbol{U}, \boldsymbol{y} \in \boldsymbol{U}$. Then $\boldsymbol{x} \in \boldsymbol{U}, \boldsymbol{g}^{\boldsymbol{n}} \boldsymbol{x} \in \boldsymbol{U}(\boldsymbol{n}=\boldsymbol{k}-\boldsymbol{l})$, which was to be proved.
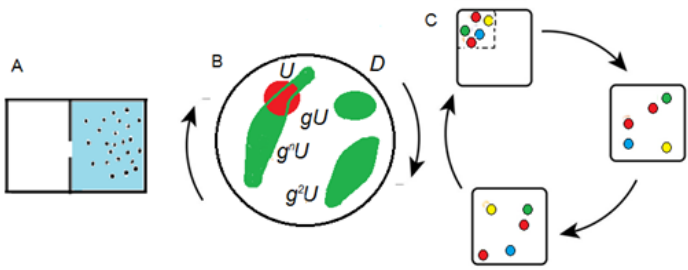

Fig. 1. A. Molecules return to the first chamber. B. Theorem on returning [1]. C. Recurrence can be demonstrated by balls in a box: when they start out in an ordered state, they will become more disordered. But at some point, they will return to the initial state - it just might take a while [2].

This theorem is one of the basic theorems of ergodic theory. The essence of it is that with a measure that preserves the mapping of space onto itself, almost every point returns to its initial neighborhood.

Zermelo proved, with the help of the Poincaré mechanical theorem, that the behavior of the ordinary kinetic model of a completely isolated gas is quasi-periodic (E. Zermelo, Annalen der Physik 293, 485, 1896).

\subsection{Verification of Poincare's theorem}

It is one of the most astonishing results of physics: when a complex system is left alone, it will return to its initial state with almost perfect precision. Gas particles, for example, chaotically swirling around in a container, will return almost exactly to their starting positions after some time. This "Poincaré Recurrence Theorem" is the foundation of modern chaos theory. For decades, scientists have investigated how this theorem can be applied to the world of quantum physics. Now, researchers at TU Vienna have successfully demonstrated a kind of "Poincaré recurrence" in a multiparticle quantum system. The results have been published in "Science" [1].

At the end of the 19th century, the French scientist Henri Poincaré studied systems which cannot be fully analyzed with perfect precision - for example solar systems consisting of many planets and asteroids, or gas particles, which keep bumping into each other. His surprising result: every state which is physically possible will be occupied by the system at some point - at least to a very good degree of approximation. If we just wait long enough, at some point all planets will form a straight line, just by coincidence. The gas particles in a box will create interesting patterns, or go back to the state in which they were when the experiment started.

A similar theorem can be proved for quantum systems. There, however, completely different rules apply: "In quantum physics, we have to come up with a completely new way of addressing this problem", says Professor Jörg Schmiedmayer from the Institute for Atomic and Subatomic Physics at TU Wien. "For very fundamental reasons, the state of a large quantum system, consisting of many particles, can never be perfectly measured. Apart from that, the particles cannot be seen as independent objects, we have to take into account that they are quantum mechanically entangled."

There have been attempts to demonstrate the effect of "Poincaré recurrence" in quantum systems, but until now this has only been possible with a very small number of particles, whose state was measured as precisely as possible. This is extremely complicated and the time it takes the system to return to its original state increases dramatically with the number of particles. Jörg Schmiedmayers team at TU Wien, however, chose a different approach: "We are not so much interested in the complete inner state of the system, which cannot be measured anyway", says Bernhard Rauer, first author of the publication. "Instead we want to ask: which quantities can we observe, that tell us something interesting about the system as a whole? And are there times at which these collective quantities return to their initial value?"

The team studied the behaviour of an ultracold gas, consisting of thousands of atoms, which is kept in place by electromagnetic fields on a chip. "There are several different quantities describing the characteristics of such a quantum gas - for example coherence lengths in the gas and correlation functions between different points in space. These parameters tell us, how closely the particles are linked by quantum mechanical effects", says Sebastian Erne, who was responsible for the theoretical calculations necessary for the project. "Our everyday intuition is not used to dealing with these quantities, but for a quantum system, they are crucial." 
By measuring such quantities, which do not refer to single particles, but characterize the system as a whole, it was indeed possible to observe the long-sought quantum recurrence. And not only that: "With our atom chip, we can even influence the time it takes the system to return to one particular state", says Jörg Schmiedmayer. "By measuring this kind of recurrence, we learn a lot about the collective dynamics of the atoms - for example about the speed of sound in the gas or about scattering phenomena of density waves."

The old question, whether quantum systems show recurrences, can finally be answered: Yes, they do - but the concept of recurrence has to be slightly redefined. Instead of trying to map out the complete inner quantum state of a system, which cannot be measured anyway, it makes more sense to concentrate on quantities which can be measured in quantum experiments. These quantities can be observed to drift away from their initial value - and to return to their initial state eventually (Fig. 1-C).

\section{Shock-wave model of an earthquake.}

\subsection{SW generation in lithosphere.}

Let's discuss the experiments on high-pressured kalicinite $\left(\mathrm{KHCO}_{3}\right)$, the mineral in which hydrogen bonds show the capacity for quantum entanglement. On compression the structure of kalicinite hydrogen bonds changes simultaneously over the whole volume of the sample (Fig. 2) resulting in the change of its unit-cell volume (or density) and correspondingly (according to our model) in the SW generation. Similar data are obtained for other minerals containing hydrogen and oxygen (nitrogen, fluorine). By data of many authors such structural phase transition of hydrogen bonds happens instantly over the large volume and this phenomenon is called a cooperativity.

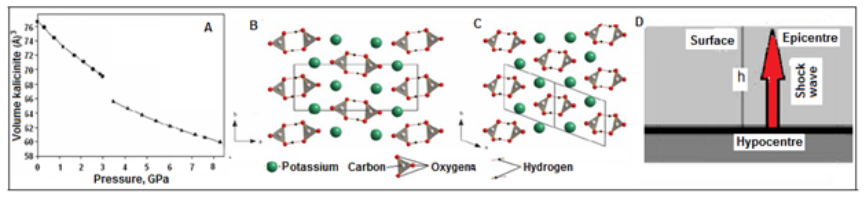

Fig. 2. The change of the unit-cell volume $(\Delta V / V=4 \%)$ for kalicinite at the pressure of $3.2 \mathrm{GPa}-\mathrm{A}$, the change of kalicinite crystal structure - B,C [9]. Shock wave generation inside the layer of lithosphere containing hydrogen bonds - D.

\subsection{Hydrogen bond.}

A hydrogen bond occurs when the electron-lacked hydrogen covalently bound to the electronegative atom (oxygen, nitrogen, fluorine) interacts with lone-electron pair of another electronegative atom in the same molecule or in the other one. Cooperativity of hydrogen bonds in water in particular is responsible for the dependence of water features on the amount of hydrogen bonds. A sizeable quantity of hydrogen bonds in ice decreases with its melting up to their almost absence in boiling water. A lack of possibility to form hydrogen bonds would result in water transformation into vapor. Hydrogen bonding also plays an important role in lithosphere properties. Shifting of electron density at the line H...O $(\mathrm{N}, \mathrm{F}$, etc.) to the electronegative atom provides hydrogen bonding which produces interatomic distances $\mathrm{O}-\mathrm{O}, \mathrm{O}-\mathrm{N}$ shorter than in hydrogen absence.

\subsection{Earthquake energy.}

We are going to estimate the energy needed for kalicinite (KHCO3) compression (see Fig 2-A). The external pressure $p$ $=3 \mathrm{GPa}$ leads to a change in the sample volume $\Delta V / V \approx 5 \%$ that is about $10^{2} \mathrm{~J} / \mathrm{cm}^{3}$. In Fig. 2-B and $\mathrm{C}$ the structural phase transition "order-disorder" is shown. In [7] the enthalpies of this transition are given for a wide range of crystals. We assume that energy of one broken hydrogen bond is about $0.5 \mathrm{eV}\left(1 \mathrm{eV} \approx 10^{-19} \mathrm{~J}\right)$. Let's consider how the energy released at fracture (fracture, rotation, etc.) of hydrogen bonds is consistent with the estimate obtained from the relation $E=p V$. In minerals under the external pressure the presence of hydrogen bonds can provide the energy necessary for the shock wave generation.

\subsection{Quantum entanglement.}

Quantum entanglement is a perspective research direction included into the list of 20 Big Scientific Ideas in "BBC Focus Special Edition, 2013". Here we try to link such notions as 'entanglement', 'decoherence', 'recoherence' and 'teleportation' with crystallization and different types of phase transitions that can occur in lithosphere and atmosphere.

Since we do not exploit measurement, we can capture 'entanglement' through collective decoherence without common environment. In our earthquake model the generation of the shock wave follows from decoherence of multiparticle quantum system and subsequent fast phase transitions. Moreover, decoherence can transform one quantum system into another which can have additional properties revealed in the form of an aftershock, teleportation of proton state and ect.

\subsection{Quantum decoherence.}

Decoherence (D) of quantum system (QS) occurs every time when its state becomes entangled with a state of the environment. Thus, 'information' of QS is imprinted in a state of its environment. It is known that D is a physical 
process accompanied with the loss of coherence because of entanglement with the environment. Moreover, after decoherence QS gets classical features reflected into environment. Under natural conditions it is impossible to isolate multiparticle QS. Therefore, open quantum systems are common. Because of entanglement with the environment a coherent state transforms into a 'classical' mixed one. Moreover, an inverse transition called recoherence is possible. For recoherence there is only need in weakening of interaction between system and environment. Since there is 'information' about quantum states imprinted in the environment, we can observe transformations of 'classical' environment induced by decoherence or recoherence. Due to the no-go theorem it is impossible to statistically verify these transformations. This idea prohibits prediction of an earthquake.

\subsection{Quantum teleportation.}

A quantum teleportation (QT) is a transition of quantum state for arbitrary long distances with the help of classical channel and separation of quantum pairs. We can a priori assume that there are entangled QS separated by a distance. Since we cannot exclude the classical interaction (classical channel), we deal with QT. Sometimes the classical channel is not obvious, for example, it is not clear how protons in hydrogen bonds of supercooled water in Arctic cyclones are classically linked with protons in hydrogen-bonded camphora of a storm-glass in Novosibirsk [8].

Another example of QT is a 'connection' between the Sumatra earthquake in 2004 and typical fluctuation of atmospheric electric field in Italy [9]. There are numerous examples of 'superluminal connection' between far distanced separated earthquakes. QT can be the only reason of such connections. It seems that sprites, jerks and particles of high energy in water saturated atmosphere are generated because of QT. By the way, here lightning bolt is a 'classical channel'.

\subsection{Accumulation of an earthquake energy.}

This procedure is usually applied in earthquakes physics. Let's do some estimates. Suppose that an earthquake occurred in a seismically active region 100 years ago. Thus, 100 years "accumulates" seismic energy has been accumulated during this period (it is not clear where and how it "accumulates"). But an earthquake occurs now after a century of "accumulation". It seems that all "accumulated" energy is expended. Since earthquakes (aftershocks) continue sometimes with energy more than that of the first shock (a foreshock) we can assume that the "accumulated" energy was expended in parts. Then there is a question who and how regulates this process.

\section{Aftershocks.}

\subsection{What do we know about aftershocks?}

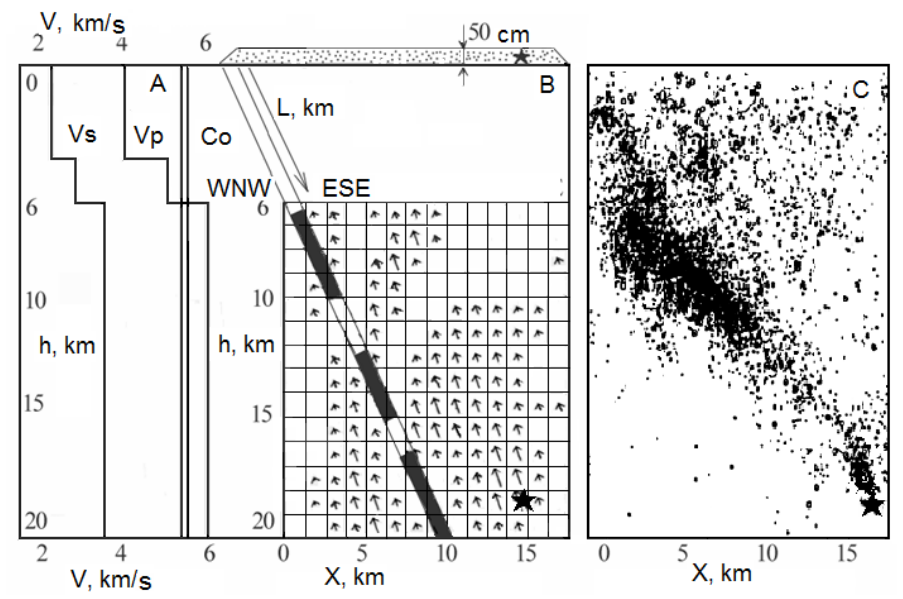

Fig. 3. Northridge earthquake [6]. A - depth distribution of $P$-, $S$-waves and $\mathrm{c}_{\mathrm{o}}$-shock wave; $\mathrm{B}$ - model of slips and the direction of the main shock; $\mathrm{C}$ - aftershocks.

Fig. 3 is related to the well-known Northridge earthquake (1994, USA). Earthquakes arising some time later the main shock and filling the space which was earlier occupied by the source or the main shock are aftershocks (Fig. 3-B). Commonly the source volume is determined from that occupied by aftershocks (Fig. 3-C). Usually the hypocenters of aftershocks, some of which are comparable in their magnitude with the main shock, are located higher than the hypocenter of the main shock. There is no understanding of aftershocks nature. The only fact revealed consists in their onset through the main shock.

\subsection{Initial observations.}

Altai (Chuya) earthquake (magnitude by the Richter scale 7.5) occurred on September 27, 2003 at 11:33 Greenwich at the south of Gorni Altai on the territory of the Kosh-Agach region. The earthquake was accompanied by numerous 
aftershocks, two of which (September 27 and October 1) were comparable in intensity to the main shock. A similar earthquake is known to occur here 100 years ago. Its traces are well preserved. Some seismologists believe that another similar strong earthquake occurred here 100 years earlier. If it turns out that a period of seismic energy accumulation is 100 years?

Why did two earthquakes similar in magnitude within the main shock occur two or three days later it? Arriving at the seismic station "Aktash" which is almost at the epicenter of aftershocks in early November we registered a series of smaller aftershocks [11]. Besides seismographic data, we recorded infrasound (Fig. 4), variations of geomagnetic field and atmosphere electricity. Magnetic and electrical data are known from other publications, but I was struck by an underground roar.

\subsection{Underground roar.}

That underground rumble (roar) preceding the earthquake shock I heard for the first time. Developing with increasing intensity the roar terminated at the time of shock. Energy class of aftershocks was small (not exceeding 10) but sitting on a chair or lying on a bed we jumped at the time of impact. It made an impression more than from a tottering wall, dishes clatter, etc. which we observed during powerful, but more distant earthquakes, occurring in Siberia and Kamchatka.

The velocities $V_{p}$ and $V_{s}$ of sound propagation, as well as SW velocity $c_{o}$ are shown in Fig. 3-A. The velocity of infrasound (underground roar) is equal to the velocity of $P$-waves $V_{p}$ and it is slightly above than $c_{o}$. The delay between the arrival of $P$ and $S W$ determines the roar duration. Immediately after the Chuya earthquake, when the seismic activity of aftershocks was high, underground roar after operator data was very intense. Sometimes no shock after an intense roar was noticed. This fact may be related to SW dissipating in matter, and its further release to the surface does not occur.

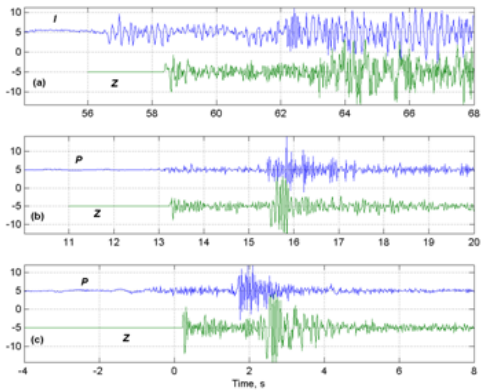

Fig. 4 Acoustic signals $I, P$ and signal $Z$ from vertical seismograph by recorded at Aktash seismic station [11] on November, 5 (a,b) and November, 8 (c).

Fig. 4 shows observations data of three aftershocks recorded at Aktash seismic station.

Seismologists know that aftershocks are practically absent after a deep earthquake, however, although rarely, this pattern is broken. After a deep $\left(564 \mathrm{~km}\right.$ ) earthquake on March 9, 1994 (with a magnitude of $M_{W}=7.6$ ) in the Tonga region the authors [12] managed to record the first series of aftershocks using eight broadband seismographs. Aftershocks (82 events) with magnitudes from 3.8 to 6.0 were attenuating after the main shock according to the power law within 42 days. Most aftershocks were localized along the vertical plane of the main shock with a maximum distance of not more than $5 \mathrm{~km}$ from it.

The seismically active medium, which includes entangled elements, has some unique properties, among which we are interested in its ability to memory. Along with the earthquakes occurrence this property manifests itself in foreshocks and aftershocks. An environment without this property cannot generate them.

\subsection{Quantum nature of aftershocks.}

The earthquake (aftershocks) time series is flicker-noise (see the Gutenberg-Richter law). In recent years the research of quantum entanglement showed that flicker-noise is inherent in multi-particles quantum (entangled) systems. Nature of earthquakes, including aftershocks follows to be quantum.

If conditions in a QS allows a full decoherence, the system is converted entirely into SW. If there is only a partial decoherence, then the second, third and next shock waves which are aftershocks may occur after formation of the first SW.

\subsection{Aftershocks energy.}

Let's try to solve the problem that is impossible to do in frames of classic physics in quantum entanglement ones. The entanglement likely terminates in changes of medium physical properties. Here is an example. Bruschi D.E., a researcher of York Centre for Quantum Technologies, Department of Physics, University of York, asked if entanglement has its own weight $[13,14]$. In this case it is interesting what role does the weight play having regard to 
acting force of gravity. Later we shall return to this not a stupid question but now we show that the entanglement changes the properties of the medium when water becomes solid, at least, not fluid through passing the current.

A laboratory experiment when two glasses filled brimful with water and set side by side is known. Electrodes applied by voltage of a few kilovolts are run into each glass. Water starts its flowing from one glass to another along the path connecting both electrodes. While the glasses are slowly pushed apart, the water continues to flow, forming a water "bridge" in which water behaves like a solid [15]. A similar state occurs in plasma of the gas discharge, into which a printer powder is added. Here, as micron-sized particles become charged and line up into a" crystal structure", plasma is called "crystal plasma". Revealed to transmit shear waves it likes a solid body. Ions formed in water and plasma are quantum entangled. "Crystal structure", similarly to that in dusty crystalline plasma, possibly accounts for a stability of the water bridge between two tanks with water under the influence of a constant electric field. If the voltage is turned off, the entanglement gets lost and the bridge breaks down. Perhaps a similar mechanism is responsible for water column in tornado behaves like a solid. And the experiment itself demonstrates the entanglement influence on physical properties of medium.

Turning to idea of Bruschi we see that he was right proposing the role of gravity in QE. Now some authors offer an idea that gravity itself is a consequence of QE of quarks and anti-quarks $[16,17]$. At its basic idea Brushi offers that entangled particles just weigh a little more than they could weigh as separate units. Thus, the gravitational perturbation becomes a function of both particle energies as expected, as well as of time during which the entanglement persists. The difference of one part at $10^{-37}$ is very small but detectable in theory. It can be registered.

These arguments imply a non-trivial conclusion: if the entanglement has its "own" energy. If so, can it be extracted and used in any way?

\subsection{Model of aftershocks.}

Let's draw Fig. 2 at Fig. 5 (left) by changing the volume of kalicinite on its density (Fig. 5 - centre). Instead of Fig. 2-D a right figure (Change in specific volume $(\Delta V / V=4 \%)$ at an external pressure of $3.2 \mathrm{GPa}$ ) corresponds to the change in density during the formation of SW-1 (asterisk - hypocenter of the main shock).

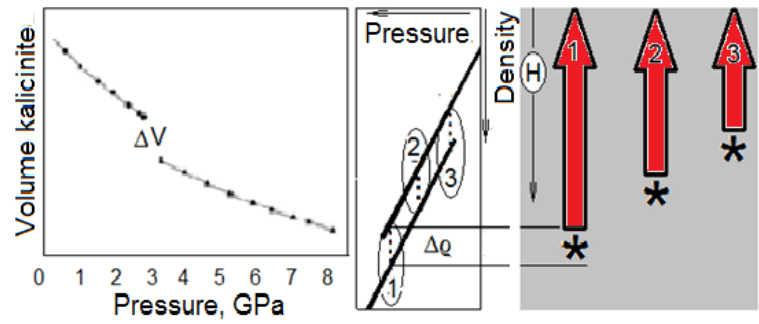

Fig. 5. Left - fig.2-A. Right - arrows point to SW by: the main shock - 1, aftershocks - 2, 3. Asterisks - hypocenters.

As it follows from Fig. 5 aftershocks formation is directed towards the Earth surface. Each shock (aftershocks follow the main shock) causes structurization of the medium above a preceding shock (2-nd above 1-st, 3-rd above 2-nd and so on). Otherwise, lithosphere pressure involves the formation of the hypocenter 1 . Hypocenters 2 and 3 occur after the lithostatic pressure is added to the pressure of the previous impact.

\subsection{Aftershocks and Poincare's theorem.}

Consider the structure of atoms of different elements and hydrogen bonds, shown in Fig. 2-B. The next Fig. 2 - C shows that there was a structural phase transition (SFP), and the substance has changed its volume. Generation of SW or an earthquake occurs here.

Imagine a situation in which the picture of Fig. 2-B is initially violated, for example, the elements of the structure do not coincide with their desired positions, or any element is missing. Then the SFP does not happen and there will be no earthquake. According to Poincare's theorem each structure "remembers" the correct location of its elements and we will strive to repeat it. Another SFP after the correct recurrence will lead to the generation of another aftershock. If this theorem is not valid, aftershocks will not arise.

\section{Shock-acoustic wave.}

\subsection{What is SAW?}

Interesting video clips of model tests of a shock-acoustic "gun" are available online. SAW generated by this device leads to the destruction of a specially constructed models of buildings, located at a distance of several dozen meters from the cannon. In the chamber of the gun SAW is generated under the bulk detonation of explosives in the form of aerosols. Nothing is discarded from the "barrel" of the gun during the shot, the SW which effects the destruction is only emitted. These experiments can be considered as the mechanistic model of the considered natural phenomenon of generation of SAW. In our case, the shock crystallization of supercooled water in the cloud plays the role of a SAW generator (detonator). 


\subsection{Shock crystallization and generation of SAW.}

Our model of SAW is alternative to the conventional one, shown in Fig.6. The model is based on experimental and theoretical studies of the shock crystallization in supercooled water. The model simultaneously solves two problems: the occurrence of hole-punch clouds and the existence of "thunder in a clear sky" phenomenon. The energy difference between the pieces of ice and drops with ice nuclei (Fig. 3-b) determines the energy of SAW. In the cloud of supercooled water there are conditions for a shock crystallization (Fig. 3-a). QS of a cloud can be quantum entangled with hydrogen bonds in the lithosphere. Here SW in a cloud is an aftershock of the main earthquake.

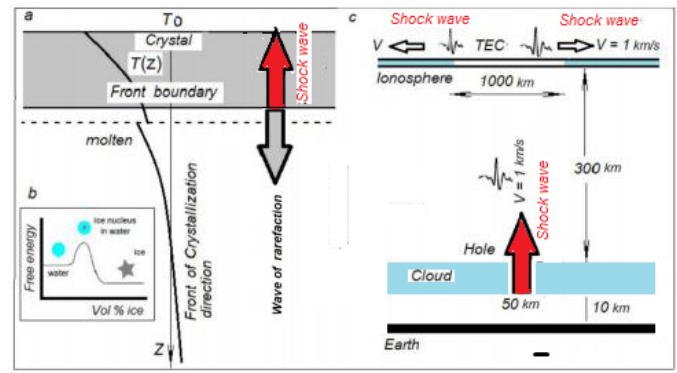

Fig. 6. Crystallization front, rarefaction waves and SAW in supercooled water of clouds - a. Comparative particle energy (Free energy) of snowflakes, water droplets with the ice nuclei and iceless water droplets - b. Reaching ionosphere SAW is propagating through it. The hole in the cloud after the shock crystallization - c. Though holes in atmosphere are well known this phenomenon is not explained.

This problem arose after my acquaintance with the book by prof. Afraymovich E. L. and Perevalova N. P. (reference in [5]), in which the authors correlate the shock wave in the ionosphere to the earthquake shock, believing that the earthquake is capable to generate SW in the ionosphere. This is not true, because the scattering rate of fragments of the destroyed soil is much less than the sound velocity in air, but the only mechanism discussed in this article is valid, if the fact of correlation between these phenomena is real (Fig. 6-C). Indeed, SAW is spreading in air and ionosphere. Unlike SW in the solid, the high-energy SAW is spreading in air without loss of energy. SAW is known to travel several times round the Earth.

The shock wave of rarefaction accompanying SAW (Fig. 6-a) throws out the ice formed by crystallization, forming a hole in a cloud. Commonly observed these holes have no generally accepted theory on their nature. The most common theory argues that holes arise due to ice formation in higher layers of clouds. Our model is not in conflict with reasons generally recognized. An ice floe moving in atmosphere parallel to the ground is shown in Fig. 7, insert. If we assume that this ice floe is formed at a hole, then the ice floes do not fall, but they have some energy which they get from the shock wave of rarefaction.

\subsection{Aftershocks and holes in clouds, "out of the blue."}

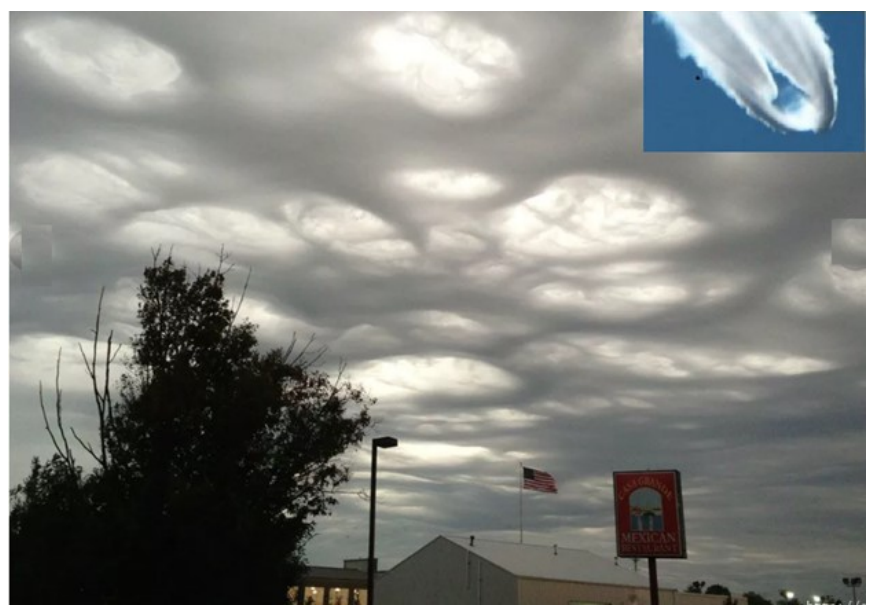

Fig. 7. Holes in clouds. The upper right corner - UFO resembles a bent ice floe, formed at generating of SAW as a wave of unloading (rarefaction). 
Perhaps SAW generates its own aftershocks which create holes in a cloud of supercooled water vapor (Fig.7). Then the question if a reverse process in which SAW generates seismic SW arises. Once academician E. P. Velikhov thought of a way to trigger earthquakes, passing a powerful electric current through the lithosphere in seismically active regions. Velikhov believed that weak earthquakes would prevent the emergence of strong ones, capable, for example, of destroying a nuclear power plant, a hydroelectric power station and other objects important for humanity. The project failed and the research I know was stopped.

Let us briefly dwell on a well-known proverb "out of the blue" which characterizes the occurrence of unpleasant situations for no apparent reason. Here it refers to SAW occurrence which cause thunder although there are no storm clouds and lightning.

\section{Conclusions.}

A fundamentally new model of aftershocks based on the shock-wave model of the earthquake and the application of the Poincare repetition (return) theorem is proposed. The action of the return theorem is confirmed by the article of Austrian physicists from the Vienna University of Technology published in Science. As it follows from the article the Poincare theorem can be formulated in such a way that complex quantum systems at some point return almost exactly to their original state. The shock-wave model of an earthquake is based on quantum entanglement of protons in hydrogen bonds of the lithosphere material. It is obvious that aftershocks are quantum phenomena with behavior regulated by this theorem.

Here we touched on an important topic concerning the origin of energy in quantum-entangled objects. I note that a challenge of "energy from nowhere" appearing in Nature arises in other problems, also related to quantum entanglement. For example, the source of electrical and mechanical energy of ball lightning (BL) in the model of BL is based on rearrangement of protons in hydrogen bonds (HB). Rearranging protons provide energy. It would seem, after protons rearranged energy was used. But no, protons continue their rearranging, and there is no clarity from where the energy emerges. Similar situations arise in physics when for some unclear reasons the fluctuation-dissipative theorem (FDT) is not fulfilled. FDT establishes a relationship between the fluctuation spectrum of physical quantities in an equilibrium dissipative medium and its generalized susceptibilities which are parameters characterizing its response to external influence. As it was shown quantum mechanics causes a violation of the fluctuation-dissipative theorem [18]. Of course, this article discusses the general case. Considered in more detail QE contribution into FDT should offer answers to our questions.

\section{References}

1. B. Rauer, S. Erne, T. Schweigler, et al., Science. 360, Is. 6386, P. 307-310. DOI: 10.1126/science.aan7938 (2018)

2. V.V. Kuznetsov, Physical mesomechanics 12(6), 87-96 (in Russian) (2009)

3. V.V. Kuznetsov, Kvantovaya magiya. 8:1. 1101-1119 (in Russian) (2011)

4. V.V. Kuznetsov, Kvantovaya magiya. 8:2 2125-2151 (in Russian) (2011)

5. V.V. Kuznetsov, Vestnik KRAUNC. Phys.-Mat. Science 3(14) 64-71. (in Russian) (2016)

6. V.V. Kuznetsov, Introduction to the physics of the hot Earth (KamGU, Petropavlovsk-Kamchatsky, 367p. (in Russian). (2008)

7. N.D. Kuznetsova, V.V. Kuznetsov, Journal of Unconventional Science 3(9) 6-12. (in Russian) (2015)

8. V. I. Arnold, Mathematical methods of classical mechanic, ISBN 978-1-4757-1693-1 (1978)

9. D.R. Allan, W.G. Marshall, C.R. Pulham, American Mineralogist. 92 1018-1025 (2007)

10. A. I. Baranov, Crystallography 48(6) 1081-1107. (in Russian) (2003)

11. V.V. Kuznetsov, S.Yu. Khomutov, Electronic scientific and information journal. 005. No 1(23).

(http://www.scgis.ru/russian/cp1251/h_dgggms/1-2005/screp-1.pdf). (in Russian).

12. D.A. Wiens, J.J. McGuire, P.JU. Shore, et al., Nature. 372 540-543 (1994)

13. D.E. Bruschi, York Centre for Quantum Technologies, Department of Physics, University of York, March 14, (2017)

14. D.E. Bruschi, York Centre for Quantum Technologies, Department of Physics, University of York, February 20, (arXiv:1701.00699v2 [gr-qc] (2017)

15. V.V. Kuznetsov, Journal of Unconventional Science 11(4) 107-110 (in Russian) (2016)

16. Sonner, J. Phys. Rev. Lett. 111, 211603. (http://arxiv.org/abs/1307.6850) (2013)

17. M. Karliner, J.L. Rosner, Nature. 551 89-91 (2017)

18. A. Shimizu, K.J. Fujikura, Stat. Mech. 024004. arXiv:1610.03161v3 [cond-mat.stat-mech] 10 Feb (2017) 